\title{
Polymer-based nanoparticulate solid dispersions prepared by a modified electrospraying process
}

\author{
Deng-Guang Yu ${ }^{1}$, Gareth R. Williams ${ }^{2}$, Xia Wang ${ }^{1 *}$, Jun-He Yang ${ }^{1}$, Xiao-Yan Li ${ }^{1}$, Wei Qian ${ }^{1}$, \\ Ying $\mathbf{L i}^{1}$ \\ ${ }^{1}$ School of Material Science \& Engineering, University of Shanghai for Science and Technology, Shanghai, China; \\ ${ }^{2}$ School of Human Sciences, Faculty of Life Sciences, London Metropolitan University, London, UK. \\ E-mail:ydg017@gmail.com, ${ }^{*}$ wangxia@usst.edu.cn
}

Received 12 October 2011; revised 24 October 2011; accepted 20 November 2011.

\begin{abstract}
A modified electrospraying process is exploited to enhance the dissolution profiles of a poorly watersoluble drug. With polyvinylpyrrolidone (PVP) as a hydrophilic polymer matrix and ketoprofen (KET) as a model drug, polymer-drug composites in the form of nanoparticles were prepared and characterized. The surface morphologies, the physical status of the drug, and the drug-polymer interactions were studied using FESEM, DSC, XRD, and ATR-FTIR. FESEM observations demonstrated that the nanoparticles gradually decreased in size from $640 \pm 350$, to $530 \pm$ $320,460 \pm 200$ and $320 \pm 160 \mathrm{~nm}$ as the KET content increased from 0 , to $9.1 \%, 16.7 \%$ and $33.3 \% \mathrm{w} / \mathrm{w}$, respectively. Results from DSC and XRD suggested that KET was distributed in the PVP matrix in an amorphous manner at the molecular level. This is thought to be due to their compatibility, arising through hydrogen bonding as demonstrated by ATRFTIR spectra. In vitro dissolution tests showed that the nanoparticles released the incorporated KET within 1 min, evidencing markedly improved dissolution over pure KET and a KET-PVP physical mixture. Electrospraying can hence offer a facile route to develop new polymer composites for biomedical applications, in particular for improving dissolution rate of poorly water-soluble drugs.
\end{abstract}

Keywords: Polymer Composites; Electrospraying; Poorly Water-Soluble Drug; Nanoparticles; Solid Dispersion; Polyvinylpyrrolidone

\section{INTRODUCTION}

The solubility behavior of poorly water-soluble drugs remains one of the most challenging aspects of formulation development $[1,2]$. Numerous advanced functional materials, new processes and technologies have been investigated in order to provide more effective and versatile ways to handle formulation issues associated with poorly water-soluble molecules [3-5]. Among them, nanosizing strategies have been widely used to enhance the dissolution and oral availability of poorly soluble drugs by enlarging the surface area of the drug powder or changing the crystalline form [6-9].

Solid dispersion (SD) is considered to be one of the most appropriate methods to improve dissolution rates and hence bioavailability of poorly water-soluble drugs. With the development of pharmaceutics, materials science (especially polymer science) and novel technologies, many new strategies and excipients have been employed for preparing SDs over the past several decades.

In traditional solvent evaporation methods for preparing SDs, the drug and carrier are first dissolved in an organic solvent or solvent mixture, and subsequently the solvent is removed. These methods differ in the ways by which the solvents are removed to solidify the products. A range of different strategies have been developed for the fast and effective removal of the solvents. These include spraying (heat or freeze spraying) and drying (fluidized bed, freeze, microwave, or vacuum drying). These methods take advantage of phase changes and exploit thermal energy, wave energy or mechanical energy to remove the organic solvents [10-15].

One concern associated with these solvent evaporation methods is that it is challenging to rapidly remove sufficient solvent from the co-precipitates, because the latter become increasingly viscous during the "drying" processes, which hinders further evaporation of the residual solvent. Furthermore, crystal lattices are often easily formed, leading to crystalline particle growth in the later stages of the drying process. This arises owing to the mobility of the drug molecules, and may compromise the properties of the SDs [16,17].

Most recently, electrical energy has been exploited to remove organic solvents directly, producing SDs in the 
form of nanofibers through an electrospinning process [18-21]. Liquids can readily interact with electric fields [22], and the electrospinning process rapidly causes drying and solidification of micro-fluid jets, producing nanosize fibers very rapidly (often on the order of $10-2$ s) [23]. Provided favorable secondary interactions exist, the physical state of the components in the liquid solutions may be propagated into the solid nanofibers. The final products have an advantageous 1D nanoscale structure and contain the poorly water-soluble drugs in an amorphous state, much sought after in SD products. Thus, the reported nanofiber SDs exhibited excellent results in enhancing the dissolution rates of poorly-water soluble drugs [18-21,23].

Based on the same principles, another process, named electrospraying, (or sometimes, improperly, electrohydrodynamic atomization (EHDA)) [24,25], has been growing in popularity owing to its ability to easily fabricate particles and thin films. Electrospraying and electrospinning are the two main EHDA techniques, and comprise unique processes in that they produce fibers and particles at the micro- and nano scale by exploiting electric forces [26-28]. A significant feature of electrospraying is its ability to generate particles with a mean diameter that can be varied between hundreds of micrometers and tens of nanometers. This is achieved by carefully controlling processing parameters such as flow rate, needle diameter, and applied voltage, as well as the chemical composition and concentration of the solution. The process is simple and straightforward.

Electrospraying has been investigated for producing materials with applications in a wide range of fields including in the pharmaceutics, ceramics, cosmetics and food industries. In the biomedical and drug delivery area, the Edirisinghe group has successfully prepared hollow microspheres, porous films, drug-loaded micro- and nanoparticles, and microbubbles using this technology [2931]. All their investigations demonstrated that electrospraying is a useful tool for developing novel bimaterials and drug delivery systems.

Polymer-based SDs have been demonstrated to be suitable for the formulation of poorly water-soluble drugs in the amorphous form, leading to enhancement of dissolution rates and bioperformance [32,33]. The polyvinylpyrrolidone (PVP) series of polymers is widely used in the pharmaceutical field for excipients, and these materials are particularly suitable for the preparation of SDs by the solvent methods. This is due to their high solubility in water (and also in many organic solvents), rapid uptake of water, and their ability to prevent the crystallization of dispersed drugs [34]. With PVP as a polymer matrix and ketoprofen (KET) as a poorly water-soluble model drug, SDs in polymer-based nanopar- ticles prepared using an electrospraying process are reported in this paper.

\section{MATERIALS AND METHODS}

\subsection{Materials}

Ketoprofen (KET, >99\%, No. 090317) was purchased from Wuhan Fortuna Chemical Co., Ltd (Wuhan, China). Polyvinylpyrrolidone K30 (PVP K30, $M_{w}=58,000$ ) was obtained from Shanghai Yunhong Pharmaceutical Aids and Technology Co. Ltd. (Shanghai, China). Anhydrous ethanol of analytical grade was purchased from the Sinopharm Chemical Reagent Co., Ltd. All other chemicals used were analytical grade, and water was distilled before use.

\subsection{Preparation of Co-Dissolving Solutions}

A series of PVP/KET co-dissolving solutions were prepared as detailed in Table 1. The solutions were degassed with a SK $5200 \mathrm{H}$ ultrasonicator $(350 \mathrm{~W}$, Shanghai Jinghong Instrument Co., Ltd. Shanghai, China) for 10 min before electrospraying.

\subsection{Electrospraying Processes}

The electrospraying processes were carried out under ambient conditions $\left(21^{\circ} \mathrm{C} \pm 3{ }^{\circ} \mathrm{C}\right.$ and relative humidity $57 \%$ $\pm 6 \%$ ). The solutions were placed in a syringe $(5 \mathrm{ml})$ with a metal needle (with outer and inner diameters of 0.7 and $0.5 \mathrm{~mm}$ respectively). A power supply (ZGF60KV/2 $\mathrm{mA}$, Shanghai Sute Co., Ltd., China) was used at a voltage of $6 \mathrm{kV}$ and the nanoparticles were collected on aluminum foil at a distance of $30 \mathrm{~cm}$. An infrared radiation heater (JD010, Shanghai Jade Gordon Machinery Co., Ltd., Shanghai, China) was used to assist the nanoparticle drying process. The flow rate was fixed at $1.0 \mathrm{ml}$ $\mathrm{h}^{-1}$ with a syringe pump (KDS100, Cole-Palmer ${ }^{\circledR}$, USA). The collected nanoparticles were placed in a DZF-6050 electric vacuum drying oven (Shanghai Laboratory Instrument Work Co. Ltd, Shanghai, China) to facilitate the removal of residual organic solvent and moisture.

\subsection{Morphology}

The surface topography of the electrosprayed nanoparti-

Table 1. Preparation conditions for the SDs.

\begin{tabular}{ccccc}
\hline & $\mathrm{P}_{0}$ & $\mathrm{P}_{1}$ & $\mathrm{P}_{2}$ & $\mathrm{P}_{3}$ \\
\hline $\mathrm{C}_{\mathrm{PVP}}{ }^{\mathrm{a}}(\mathrm{w} / \mathrm{v} \%)$ & 10 & 10 & 10 & 10 \\
$\mathrm{C}_{\mathrm{KET}}{ }^{\mathrm{b}}(\mathrm{w} / \mathrm{v} \%)$ & 0 & 1 & 2 & 5 \\
$\mathrm{P}_{\mathrm{KET}}{ }^{\mathrm{c}}(\mathrm{w} / \mathrm{w} \%)$ & 0 & 9.1 & 16.7 & 33.3 \\
\hline
\end{tabular}

${ }^{\mathrm{a}} \mathrm{C}_{\mathrm{PVP}}$ : concentration of PVP in solutions; ${ }^{\mathrm{b}} \mathrm{C}_{\mathrm{KET}}$ : concentration of KET in spinning solutions; ${ }^{c} \mathrm{P}_{\mathrm{KET}}$ : percentage $\mathrm{KET}$ in the nanofibers, $\mathrm{P}_{\mathrm{KET}}=\mathrm{C}_{\mathrm{KET}}$ / $\left(\mathrm{C}_{\mathrm{PVP}}+\mathrm{C}_{\mathrm{KET}}\right) \times 100 \%$. 
cles, the raw KET and PVP particles were observed under cross-polarized light using an XP-700 polarized optical microscope (Shanghai Changfang Optical Instrument Co., Ltd). Samples of electrosprayed nanoparticles for microscopy were prepared by collecting them for 10 min using glass microscope slides fixed on an earthed electrode of aluminum foil for direct observation.

The morphologies of raw KET particles and electrosprayed nanoparticles were also assessed using a S-4800 field emission scanning electron microscope (FESEM, Hitachi, Tokyo, Japan). The average diameter was determined from FESEM images by measuring the diameters of over 100 nanoparticles, using the Image J software (National Institutes of Health, Bethesda, USA). Prior to examination, samples were carbon sputtercoated under argon. Pictures were then taken at an excitation voltage of $5 \mathrm{kV}$.

\subsection{Stability of KET during the Electrospraying Process}

${ }^{1} \mathrm{H}$ NMR spectra were recorded to investigate the chemical stability of the loaded KET during the electrospraying process, with deuterated dimethylsulfoxide (DMSO-d6) employed as solvent. Spectra were recorded on a Bruker DRX $400 \mathrm{MHz}$ NMR spectrometer.

\subsection{Physical Status of the Components and Their Interactions}

Differential scanning calorimetry (DSC) analyses were carried out using an MDSC 2910 differential scanning calorimeter (TA Instruments Co., USA). Sealed samples were heated at $10^{\circ} \mathrm{C} \cdot \mathrm{min}^{-1}$ from 20 to $250^{\circ} \mathrm{C}$ under a flow of nitrogen gas $\left(40 \mathrm{ml} \cdot \mathrm{min}^{-1}\right)$.

$\mathrm{X}$-ray diffraction patterns (XRD) were obtained on a D/Max-BR diffractometer (Rigaku, Tokyo, Japan) with $\mathrm{Cu} \mathrm{K} \alpha$ radiation over the $2 \theta$ range $5^{\circ}-60^{\circ}$ at $40 \mathrm{mV}$ and $30 \mathrm{~mA}$.

Attenuated total reflectance Fourier transform infrared (ATR-FTIR) analysis was carried out on a NicoletNexus 670 FTIR spectrometer (Nicolet Instrument Corporation, Madison, USA) over the range $500-4000 \mathrm{~cm}^{-1}$ and at a resolution of $2 \mathrm{~cm}^{-1}$.

\subsection{In Vitro Dissolution Tests}

In vitro dissolution studies were conducted according to the Chinese Pharmacopoeia (2005 Ed.). Method II, the paddle method, was performed using a RCZ-8A dissolution apparatus (Tianjin University Radio Factory, China) at $50 \mathrm{rpm}$. Samples containing about $50 \mathrm{mg}$ of KET were put in $600 \mathrm{~mL}$ of Phosphate buffer solution (PBS, $\mathrm{pH} 6.8,0.1 \mathrm{M})$ at $37^{\circ} \mathrm{C}$, with sink conditions $C<0.2 C_{\mathrm{s}}$. At predetermined time intervals, $5.0 \mathrm{~mL}$ aliquots were withdrawn from the dissolution medium and replaced immediately with fresh medium to maintain a constant volume, by means of injectors. After filtration and appropriate dilution with PBS, the sample solutions were analyzed at $260 \mathrm{~nm}$ on a UV spectrophotometer (Unico Instrument Co. Ltd., Shanghai, China). The amount of KET present in the sample was calculated with the help of an appropriate calibration curve constructed from the Chinese Pharmacopeia (2005 Ed). All measurements were carried out six times and the amount of KET dissolved at specified time points was plotted as percentage released versus time (seconds). Raw KET particles $(<100 \mu \mathrm{m})$ and a physical mixture (PM) of KET and PVP with a weight ratio of 1:10 were used as controls.

\section{RESULTS AND DISCUSSION}

\subsection{Electrospraying process}

As shown in Figure 1(a), akin to an electrospinning system, a standard electrospraying system comprises four major components: a high-voltage power supply, an earthed collector, a fluid driving device (syringe pump), and a capillary for introducing the sprayed fluid [25,35].

In a typical electrospraying process, a liquid is fed to a metal capillary at the end of which a droplet is formed. When the droplet is exposed to a strong electric field, a charge is induced on its surface. Provided the liquid has sufficient electrical conductivity, there will be a range of combinations of the liquid flow rate and the applied voltage for which the drop will assume a conical shape (the Taylor cone). At the apex of this cone, a narrow jet is formed (cone-jet mode) which subsequently breaks up into fine droplets (Figures 1(b) and (d)).

Electrospraying first generates near-monodisperse droplets whose size can be varied between a few to hundreds of micrometers. Later, the droplets rapidly shrink due to the fast evaporation of solvents resulting from the Coulombic explosion (Figures 1(c) and (d)). The huge surface areas of the micro-droplets provide the possibility for complete of the solvents and the solidification of products. The facile interactions of electrons with fluid solvents accelerate their evaporation. If the solvent in the droplets does not evaporate effectively, they will form thin films on the collector. If the solvent evaporates well, the droplets shrink, and finally solid particles are formed.

Thus, the circumstance of solvent removal from the droplets is key to determining the format of the final products. Exhausting all the solvents is important for the preparation of electrosprayed particles. Different methods have been employed for this, such as using a cross flow of nitrogen, air or a liquid bath to extract sufficient solvent to coagulate the particles $[36,37]$. Here, an infrared radiation heater was used to facilitate the evaporation of solvents and the solidification of the nanoparticles. 
(a)

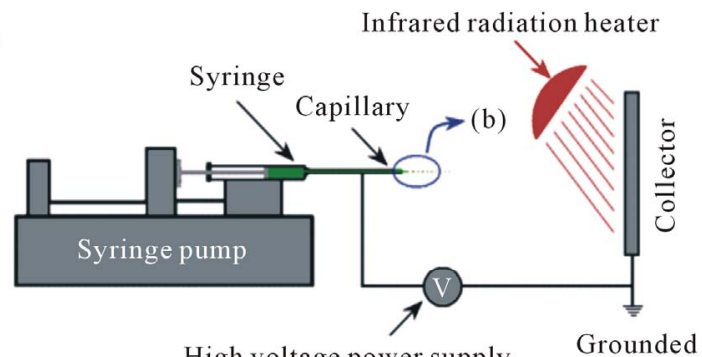

High voltage power supply

(b)

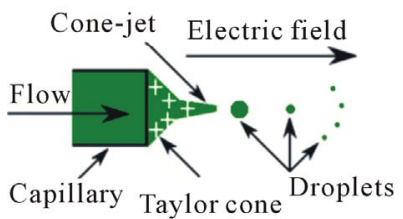

(c)

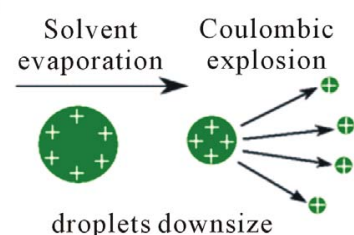

(d)

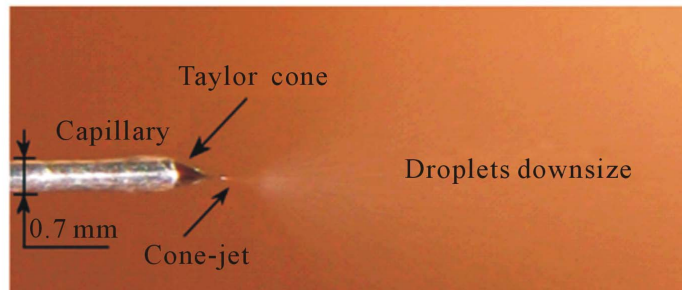

Figure 1. Electrospraying process and atomization mechanisms: (a) a schematic diagram of the electrospraying process; (b) the formation of the Taylor cone and cone-jet at the spraying head; (c) Coulombic fission of the charged liquid droplet into smaller droplets; (d) a photograph of the actual electrospraying process taken in situ.

\subsection{Morphology}

The surface morphologies of the raw KET and PVP K30 particles, as observed under cross-polarized light, are shown in Figures 2(a) and (c). Raw KET particles, a white powder to the naked eye, are seen to comprise colorful polychromatic crystals (Figure 2(a)). They naturally settled in the form of clumps, and revealed a rough surface, as indicated by the FESEM images in Figure 2(b). PVP, an amorphous polymer, was semi-transparent and its particles were spherical in shape (Figure 2(c)).

When the electrosprayed P3 nanoparticles (33.3\% KET w/w) were observed under cross-polarized light, the image was black. The particles neither gave bright color as the KET particles did, nor showed a semitransparent property like the PVP particles. This suggests that PVP and KET might have formed composites after the electrospraying process, losing their original physical status. Photographs of P3 nanoparticles under non-polarized light with a magnification of $40 \times 16$ are given in Figure 2(d).
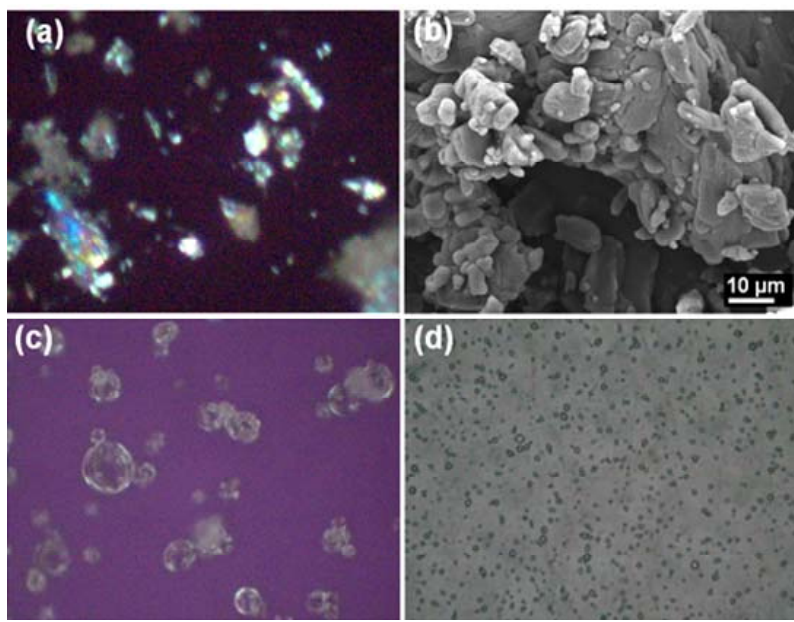

Figure 2. Morphologies of the raw materials and electrosprayed P3 nanoparticles: (a) Optical photographs of KET taken under polarized light with a magnification of $10 \times 11$; (b) FESEM images of KET; (c) Optical photographs of PVP taken under polarized light with a magnification of $10 \times 11$; (d) Optical photographs of P3 nanoparticles taken under non-polarized light with a magnification of $40 \times 16$.

FESEM images of the electrosprayed nanoparticles are depicted in Figure 3. As the KET content in the co-dissolving increased, the size of nanoparticles decreased. Particle sizes were $640 \pm 350(\mathrm{P} 0), 530 \pm 320$ (P1), $460 \pm 200(\mathrm{P} 2)$ and $320 \pm 160 \mathrm{~nm}(\mathrm{P} 3)$, with KET contents of $0,9.1 \%, 16.7 \%$ and $33.3 \% \mathrm{w} / \mathrm{w}$, respectively. The reasons for this are likely to be: 1) the presence of KET in the solutions increased their conductivities, and thus improved atomization effectiveness; 2) KET could interact with PVP molecules through hydrogen bonds, resulting in more compact nanoparticles. This idea is supported by the fact that all the P0 nanoparticles are flat with depressions, while all the $\mathrm{P} 3$ particles are spherical. Most of the P1 and P2 particles are spherical, with some still exhibiting depressions as indicated by the inset of Figures 3(b) and (c). The increase of KET in the solution additionally causes tails to form on the $\mathrm{P} 2$ nanoparticles, and short linear nanofibers among the $\mathrm{P} 3$ particles, suggesting that hydrogen bonds between PVP and KET enhance the entanglements of PVP molecules to improve the electrospinnability.

\subsection{Chemical Stability}

Electrospraying is well known for applications in mass spectroscopy $[27,28]$. For its application in the pharmaceutical field, it is crucial that the pharmaceutically active ingredients are chemically stable during the high electrical energy process. Only if integrity and stability is established can the potential advantages of electrosprayed products for developing drug delivery systems be explored. 

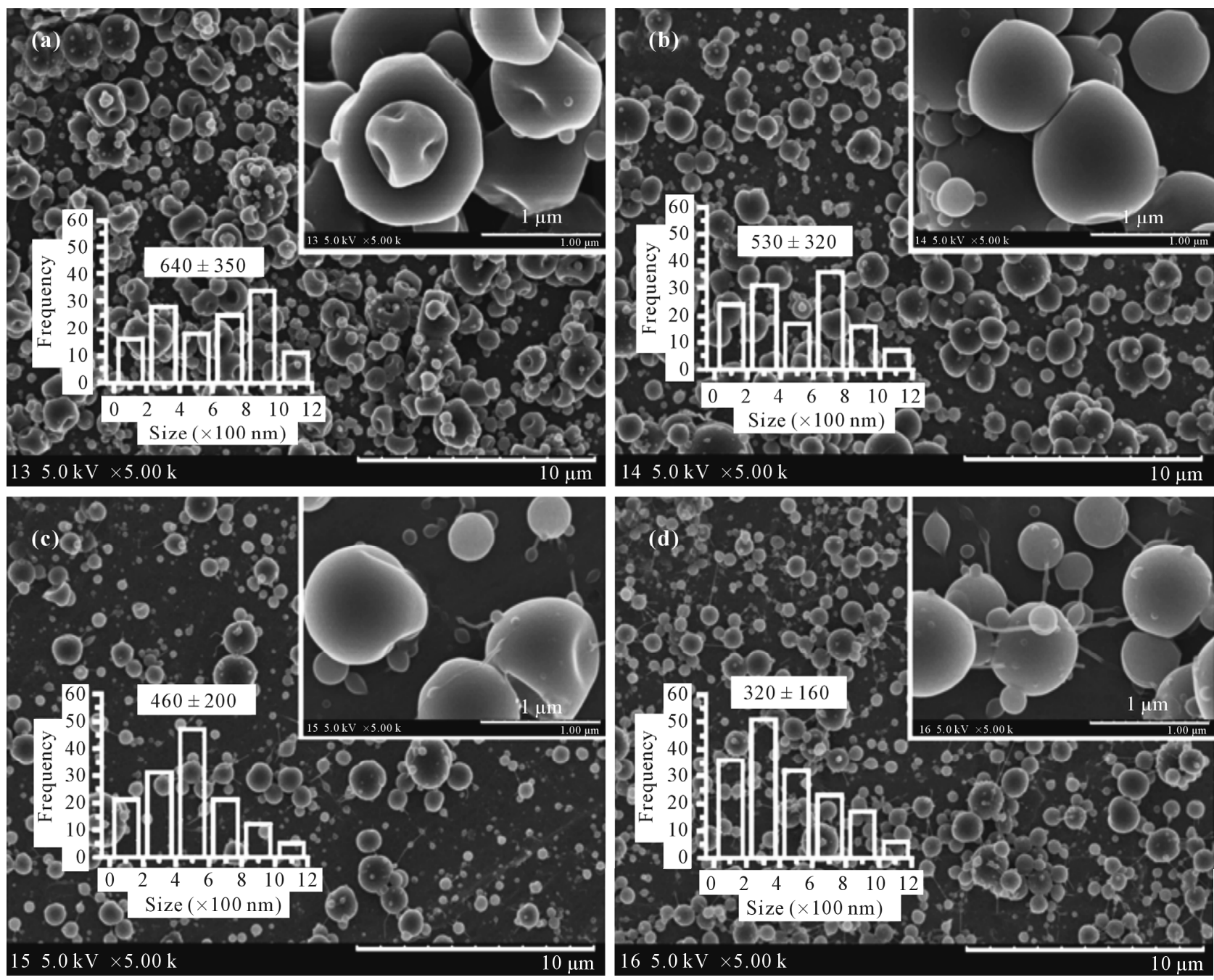

Figure 3. FESEM images of the electrosprayed nanoparticles: (a) P0, (b) P1, (c) P2, (d) P3.

To verify KET molecular integrity after the electrospraying process, drug-loaded electrosprayed nanoparticles were dissolved in DMSO- $\mathrm{d}_{6}$ and the resulting solutions were analyzed by ${ }^{1} \mathrm{H}$ NMR. Solutions of both drug-free PVP electrosprayed particles and pure KET in DMSO- $\mathrm{d}_{6}$ were used as references. Figure 4 shows the ${ }^{1} \mathrm{H}$ NMR spectra obtained from a solution containing 5 $w t \%$ of solute. The chemical integrity of the KET was maintained after the electrospraying process, as the full set of peaks corresponding to both PVP and KET could be observed in the ${ }^{1} \mathrm{H}$ NMR spectrum of the drug-loaded particles.

\subsection{Physical Status and Polymer-Drug Interactions}

In the applications of a new technology in the pharmaceutical field, it is often desirable if the process can alter the physical status of drug to the favorable nanocrystalline, amorphous or solid solution phases. To further in-

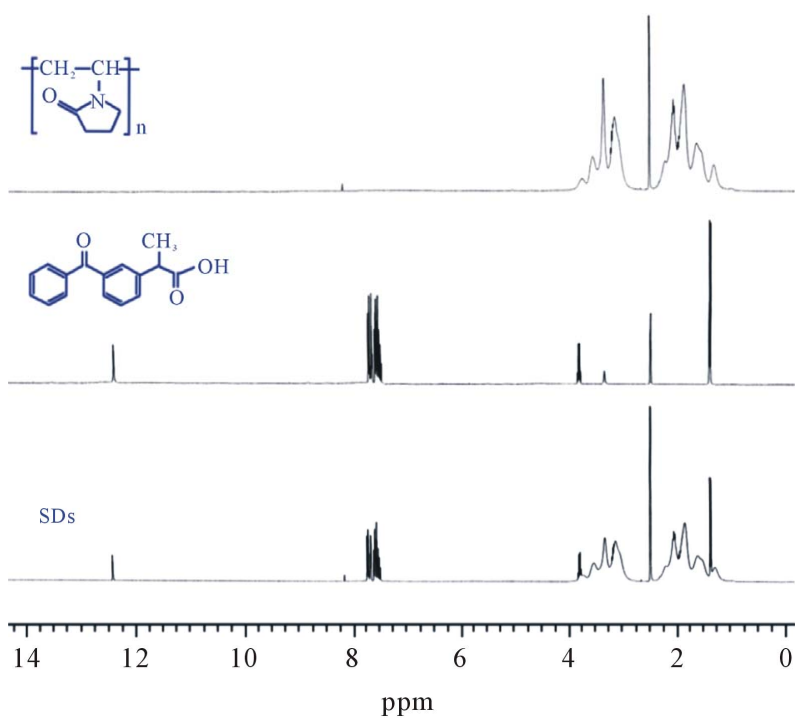

Figure 4. ${ }^{1} \mathrm{H}$ NMR spectra of SDs, PVP and KET in 5 $w t \%$ solutions in DMSO- $\mathrm{d}_{6}$. 
vestigate the physical status of KET in the electrosprayed composite nanoparticles, DSC and XRD analyses were conducted.

The DSC curve of pure KET (Figure 5) exhibited a single endothermic response corresponding to the melting of the ketoprofen at $95.7^{\circ} \mathrm{C}\left(\Delta H_{f} 114.7 \mathrm{~J} / \mathrm{g}\right)$. Being comprised solely of the amorphous polymer PVP K30, P0 did not show any fusion peak or phase transition apart from a broad endotherm due to dehydration, which lies between $60^{\circ} \mathrm{C}-120^{\circ} \mathrm{C}$ with its peak at $85.4^{\circ} \mathrm{C}$.

DSC thermograms of the KET-loaded electrosprayed nanoparticles P1, P2 and P3 did not show the KET melting peak, but instead included a broad endotherm ranging from $40^{\circ} \mathrm{C}$ to $110^{\circ} \mathrm{C}$ with transition temperatures lower than pure PVP. These observations suggested that KET was no longer present as a crystalline material in the nanoparticles, but had been converted into an amorphous state.

The XRD patterns of pure KET, $\mathrm{P} 0$ and $\mathrm{P} 1$ to $\mathrm{P} 3$ are compared in Figure 6. The presence of numerous distinct reflections indicated that pure KET comprised a crystalline material. The pure PVP diffractogram exhibits a diffuse background pattern with two diffraction halos, which means that the polymer is amorphous. In the diffractograms of P1 to P3, none of the characteristic diffraction peaks of KET were present. This confirms the loss of the crystalline KET structure in the electrosprayed nanoparticles. The results of XRD concurred with the morphological observations and DSC, confirming that KET existed in the electrosprayed nanoparticles as SDs, very different from its form in the pure drug.

The compatibility between the active pharmaceutical ingredients and the polymer carrier is essential for producing high quality products, for physical stability during long time periods of storage, and sometimes for a trouble-free processing procedure. Often, second-order interactions such as hydrogen bonding, electrostatic interactions, and hydrophobic interactions between compounds in a formulation improve their compatibility.

Thus, the interactions between PVP and KET were investigated using ATR-FTIR. The spectrum of pure PVP showed intense bands at $2955 \mathrm{~cm}^{-1}$ (C-H stretch) and $1661 \mathrm{~cm}^{-1}(\mathrm{C}=\mathrm{O})$ (Figure 7). Two well defined, sharp peaks are visible for pure crystalline KET: one at $1697 \mathrm{~cm}^{-1}$, representing the stretching vibration of the carbonyl group in the dimeric carboxylic acid, and the other at $1655 \mathrm{~cm}^{-1}$ due to the stretching vibration of the carbonyl group [38]. The former absorption arises because KET molecules are bound together in dimers in the crystalline form. However, this peak is not visible in the in the spectra of $\mathrm{P} 1$ and $\mathrm{P} 2$, indicating the breakage of the interaction between the KET molecules and the formation of a hydrogen bond between the PVP carbonyl group and

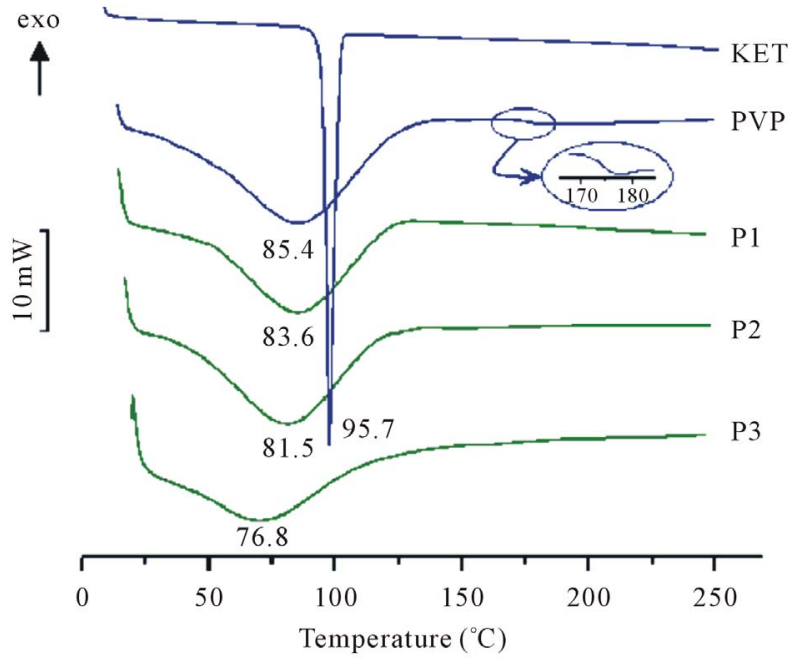

Figure 5. DSC curves.

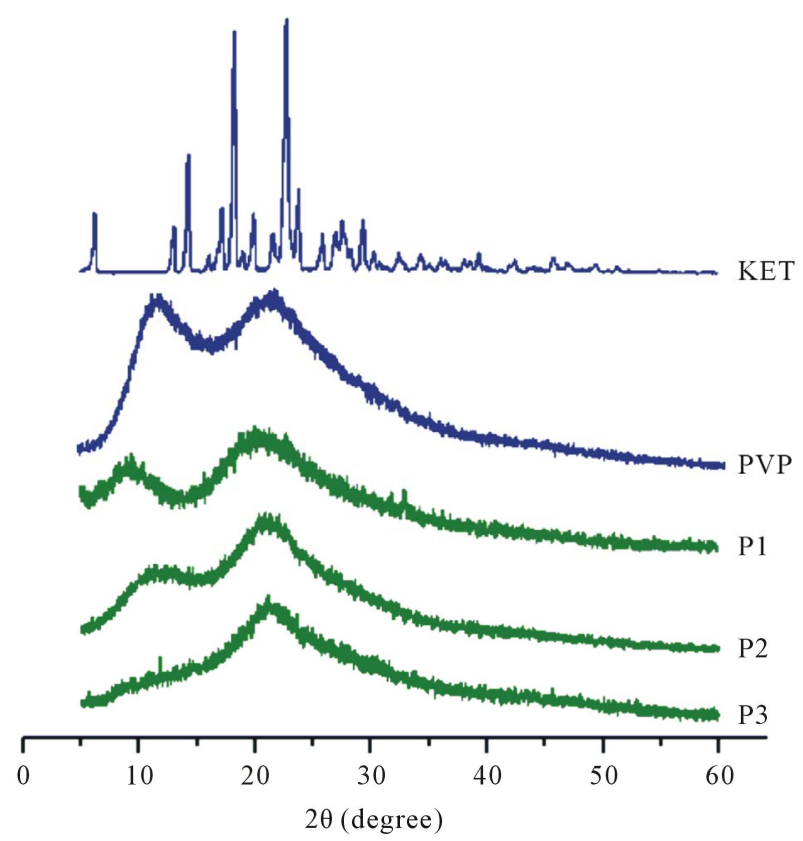

Figure 6. X-ray diffraction patterns.

the $\mathrm{COOH}$ group of the KET molecule [39]. KET molecules, when interacting with the polymer, are less likely to form the dimers which are essential for crystal lattice formation [40]. However, there is a tiny peak in the P3 spectrum at $1695 \mathrm{~cm}^{-1}$. This suggests that there is some crystalline KET in the nanoparticles when the KET content is raised to a level of $33.3 \% \mathrm{w} / \mathrm{w}$, which could not be detected by DSC and XRD. Although there may be a secondary mechanism involving electrostatic/hydrophobic interactions through the KET benzene ring [41], it is mainly the hydrogen bonding interactions that are responsible for both for KET crystallization and its inhibition. These interactions must have been helpful for the 


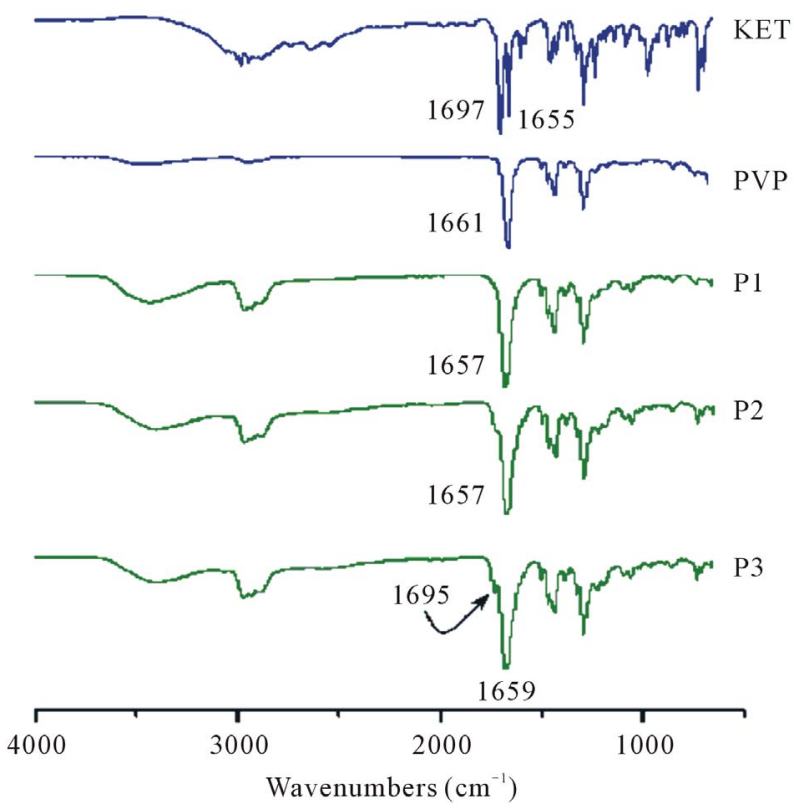

Figure 7. ATR-FTIR spectra.

stability of drug SDs in the electrosprayed nanoparticles, and could also confer a dissolution rate advantage for KET.

\subsection{In Vitro Dissolution Tests}

The in vitro dissolution profiles of the pure KET particles, a physical mixture of KET and PVP (PM), and P1 to $\mathrm{P} 3$ (Figure 8) demonstrated a marked improvement of the dissolution rate of KET in the nanoparticles compared to the pure drug and PM. In the first minute, almost all the KET in P1 and P2 and 93.4\% in P3 was free in the dissolution media. In contrast, only $0.4 \%$ of the drug was freed from the pure drug particles and $4.3 \%$ of KET from the PM inside 1 minute.

The favorable dissolution profiles of KET in the nanoparticles can be partially ascribed to their high surface area and the excellent wettability of PVP. However, the most important reason is that KET co-exists with PVP in the nanoparticles in an amorphous and highly homogeneous state. Transformation of the crystalline drug to the amorphous molecular state upon solid dispersion formulation increases the dissolution rate, since no lattice structure has to be broken down for dissolution to take place. During the rapid dissolution process of the nanoparticles' PVP matrix, the embedded KET in the nanoparticles simultaneously dissolved out and was freed into the dissolution medium. Thus, drug release from the nanoparticles was via a "polymer-controlled" mechanism. The synergistic effects of nanosizing of the particles, and forming amorphous composites of the drug with a hydrophilic polymer matrix endowed this novel type of SD with the marked improvement in dissolution

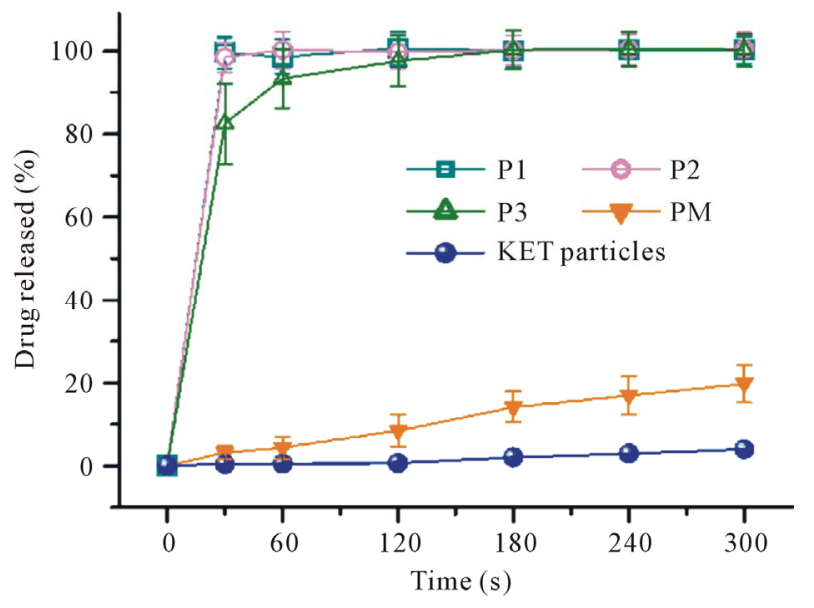

Figure 8. In vitro dissolution profiles of KET.

rate.

\section{CONCLUSIONS}

Using PVP as a hydrophilic polymer matrix and KET as an example of a poorly water-soluble drug, SDs in the form of nanoparticles have been successfully prepared using a single fluid electrospraying process. An infrared radiation heater was used to assist the evaporation of the solvents and the solidification of the nanoparticles. As the content of KET in the nanoparticles increased, particle size gradually decreased and their morphologies became spherical, with tails or nanofibers among the particles. XRD and DSC results demonstrated that KET was present in the nanoparticles in an amorphous state. ATRFTIR showed that the main interaction between KET and PVP is hydrogen bonding. In vitro tests proved that the electrosprayed nanoparticles released almost all the embedded KET within one minute, showing markedly improved dissolution properties. By virtue of electrical forces, the simple one-step electrospraying process provides a viable route to prepare drug-polymer composites and for improving the dissolution profiles of poorly water-soluble drugs.

Nanotechnology is now on the way to making a very significant impact in biotechnology, pharmaceutical and medical diagnostic sciences. Nano-packaging of medicines can increase drug efficacy, specificity, tolerability, and therapeutic index. Polymer-based nanoscale drug delivery systems (DDS) can potentially further protect drugs from degradation, regulate drug release profiles and reduce toxicity or side effects [42-44]. The research reported here shows the facile nanopackaging of a poorly water-soluble drug in a polymer carrier using electrospraying. It not only demonstrates the production of SD in nanoparticles, but may also be exploited to prepare DDS for sustained drug release profiles and targeted drug delivery with a change of polymer matrix. Com- 
pared to the electrospinning process and electrospun nanofibers, electrospraying and its corresponding nanoparticles is likely to have more diverse applications in the pharmaceutical field. All polymer excipients can be electrosprayed into micro- or nano-particles with active pharmaceutical ingredients, provided suitable solvents are employed. In contrast, only limited polymer excipients with appropriate molecular weights have good electrospinnability.

\section{ACKNOWLEDGEMENTS}

We would like to thank the scientific starting funds for young teachers of University of Shanghai for Science and Technology (No. 10-00310-001) and Grant 10JC1411700 from the Science and Technology Commission of Shanghai Municipality.

\section{REFERENCES}

[1] Vasconcelos, T., Sarmento, B. and Costa, P. (2007) Solid dispersions as strategy to improve oral bioavailability of poor water-soluble drugs. Drug Discovery Today, 12, 10681075. doi:10.1016/j.drudis.2007.09.005

[2] Nagy, Zs. K., Nyú, K., Wagner, I., Molnár, K. and Marosi, Gy. (2010) Electrospun water soluble polymer mat for ultrafast release of Donepezil HCl. eXPRESS Polymer Letters, 4, 763-772.

[3] Blagden, N., De Matas, M., Gavan, P. T. and York, P. (2007) Crystal engineering of active pharmaceutical ingredients to improve solubility and dissolution rates. $A d$ vanced Drug Delivery Reviews, 59, 617-630. doi:10.1016/i.addr.2007.05.011

[4] Yu, D.G., Branford-White, C., White, K., Annie Bligh, S.W., Williams, G., Zhu, L. M. and Chatterton, N.P. (2011) Self-assembled liposomes from amphiphilic electrospun nanofibers. Soft Matter, 7, 8239-8247. doi:10.1039/c1sm05961k

[5] Rasenack, N. and Muller, B.W. (2002) Dissolution rate enhancement by in situ micronization of poorly water-soluble drugs. Pharmaceutical Research, 19, 18941900. doi:10.1023/A:1021410028371

[6] Farokhzad, O.C. and Langer, R. (2009) Impact of nanotechnology on drug delivery. ACS Nano, 2009, 3, 16-20. doi:10.1021/nn900002m

[7] Moshfeghi, A.A. and Peyman, G.A. (2005) Micro- and nanoparticulates. Advanced Drug Delivery Reviews, 57, 2047 2052. doi:10.1016/j.addr.2005.09.006

[8] Nagy, Z.K., Balogh, A., Vajna, B., Farkas, A., Patyi, G., Kramarics, Á. and Marosi, G. (2011) Comparison of electrospun and extruded soluplus ${ }^{\mathbb{B}}$-based solid dosage forms of improved dissolution. Journal of Pharmaceutical Sciences, 100, 322-332. doi:10.1002/jps.22731

[9] Farokhzad, O.C. (2008) Nanotechnology for drug delivery: the perfect partnership. Expert Opinion on Drug Delivery, 5, 927-929. doi:10.1517/17425247.5.9.927

[10] Fini, A., Cavallari, C., Ospitali F. and Gonzalez-Rodriguez, M.L. (2011) Theophylline-loaded compritol microspheres prepared by ultrasound-assisted atomization. Journal of Pharmaceutical Sciences, 100, 743-757. doi:10.1002/jps.22312
[11] Al-Obaidi, H., Brocchini, S. and Buckton, G. (2009) Anomalous properties of spray dried solid dispersions. Journal of Pharmaceutical Sciences, 98, 4724-4737. doi:10.1002/jps.21782

[12] Moneghini, M., Bellich, B., Baxa, P. and Princivalle, F. (2008) Microwave generated solid dispersions containing ibuprofen. International Journal of Pharmaceutics, 361, 125-130. doi:10.1016/i.ijpharm.2008.05.026

[13] Patel, R. and Patel, P. (2008) Preparation, characterization, and dissolution behavior of a solid dispersion of simvastatin with polyethylene glycol 4000 and polyvinylpyrrolidone K30. Journal of Dispersion Science and Technology, 29, 193-204. doi:10.1080/01932690701706946

[14] Lee, J. and Yu, C. (2006) Critical freezing rate in freeze drying nanocrystal dispersions. Journal of Controlled Release, 111, 185-192. doi:10.1016/j.jconrel.2005.12.003

[15] Pasquali, I., Bettini, R. and Giordano, F. (2008) Supercritical fluid technologies: An innovative approach for manipulating the solid-state of pharmaceuticals. Advanced Drug Delivery Reviews, 60, 399-410. doi:10.1016/j.addr.2007.08.030

[16] Sun, N.Y., Wei, X.L., Wu, B. J., Chen, J., Lu Y. and Wu, W. (2007) Enhanced dissolution of silymarin/polyvinylpyrrolidone solid dispersion pellets prepared by a onestep fluid-bed coating technique. Powder Technology, 182, 72-80. doi:10.1016/j.powtec.2007.05.029

[17] Ivanisevic, I. (2010) Physical stability studies of miscible amorphous solid dispersions. Journal of Pharmaceutical Sciences, 99, 4005-4012. doi:10.1002/jps.22247

[18] Yu, D. G., Shen, X.X., Branford-White, C., White, K., Bligh, S.W.A. and Zhu, L.M. (2009) Oral fast-dissolving drug delivery membranes prepared from electrospun PVP ultrafine fibers. Nanotechnology, 20, 055104(9).

[19] Yu, D.G., White, K, Yang J.H., Wang, X., Qian, W. and Li, Y. (2012) PVP nanofibers prepared using co-axial electrospinning with salt solution as sheath fluid. Materials Letters, 67, 78-80. doi:10.1016/j.matlet.2011.09.035

[20] Yu, D.G., Lu, P., Branford-White, C., Yang, J.H. and Wang, X. (2011) Polyacrylonitrile nanofibers prepared using coaxial electrospinning with $\mathrm{LiCl}$ solution as sheath fluid. Nanotechnology, 22, 435301(7).

[21] Yu, D.G., Yang, J.M., Branford-White, C., Lu P., Zhang, L. and Zhu L.M. (2010) Third generation solid dispersions of ferulic acid in electrospun composite nanofibers. International Journal of Pharmaceutics, 400, 158-164.

[22] Salata, O.V. (2005) Tools of nanotechnology: Electrospray. Current Nanoscience, 1, 25-33. doi:10.2174/1573413052953192

[23] Yu, D.G., Gao L.D., White, K., Branford-White, C., Lu, W.Y. and Zhu, L.M. (2010) Multicomponent composite nanofibers electrospun from hot aqueous solutions of a poorly soluble drug. Pharmaceutical Research, 27, 24662477. doi:10.1007/s11095-010-0239-y

[24] Yu, D.G., Williams, G.R., Yang, J.H., Wang, X., Yang, J.M. and Li, X.Y. (2011) Solid lipid nanoparticles self-assembled from electrosprayed polymer-based micoparticles. Journal of Material Chemistry, 21, 15957-15961. doi:10.1039/c1jm12720a

[25] Chakraborty, S., Liao, I. C., Adler, A. and Leong, K.W. (2009) Electrohydrodynamics: Afacile technique to fabricate drug delivery systems. Advanced Drug Delivery Reviews, 61, 1043-1054. doi:10.1016/j.addr.2009.07.013 
[26] Wu, Y. and Clark, R.L. (2008) Electrohydrodynamic atomization: A versatile process for preparing materials for biomedical applications. Journal of Biomaterials Science, Polymer Edition, 19, 573-601. doi:10.1163/156856208784089616

[27] Heikkilä, P. and Harlin, A. (2009) Electrospinning of polyacrylonitrile (PAN) solution:Effect of conductive additive and filler on the process. eXPRESS Polymer Letters, 3, 437-445.

[28] Ding, L., Lee, T. and Wang, C.H. (2005) Fabrication of monodispersed taxol-loaded particles using electrohydrodynamic atomization. Journal of Controlled Release, 102, 395-413. doi:10.1016/j.jconrel.2004.10.011

[29] Chang, M.W., Stride, E. and Edirisinghe, M. (2010) A new method for the preparation of monoporous hollow microspheres. Langmuir, 26, 5115-5121. doi:10.1021/la903592s

[30] Enayati, M., Ahmad, Z., Stride, E. and Edirisinghe, M. (2010) One step electrohydrodynamic production of drugloaded micro- and nano-particles. Journal of the Royal Society Interface, 7, 667-675. doi:10.1098/rsif.2009.0348

[31] Stride, E. and Edirisinghe, M. (2008) Novel microbubble preparation technologies. Soft Matter, 4, 2350-2359.

[32] Qian, F., Huang, J. and Hussain, M.A. (2010) Drugpolymer solubility and miscibility: Stability consideration and practical challenges in amorphous solid dispersion development. Journal of Pharmaceutical Sciences, 99, 2941-2947. doi:10.1002/jps.22074

[33] Guedes, F. L., de Oliveira, B.G., Hernandes, M.Z., De Simone, C.A., Veiga, F.J., de Lima, Mdo C., Pitta, I.R., Galdino, S.L. and Neto, P.J. (2011) Solid dispersions of imidazolidinedione by PEG and PVP polymers with potential antischistosomal activities. AAPS PharmSciTech, 12, 401-410. doi:10.1208/s12249-010-9556-Z

[34] Wu, K., Li, J., Wang, W. and Winstead, D.A. (2009) Formation and characterization of solid dispersions of piroxicam and polyvinylpyrrolidone using spray drying and precipitation with compressed antisolvent. Journal of Pharmaceutical Sciences, 98, 2422-2431. doi:10.1002/jps.21598

[35] Wang, C., Chien, H. S., Hsu, C. H., Wang, Y.C., Wang, C.T. and Lu, H.A. (2007) Electrospinning of polyacry- lonitrile solutions at elevated temperatures. Macromolecules, 40, 7973-7983. doi:10.1021/ma070508n

[36] Xie, J., Lim, L.K., Phua, Y., Hua, J. and Wang, C.H. (2006) Electrohydrodynamic atomization for biodegradable polymeric particle production. Journal of Colloid and Interface Science, 302, 103-112. doi:10.1016/i.jcis.2006.06.037

[37] Pareta, R. and Edirisinghe, M.J. (2006) A novel method for the preparation of biodegradable microspheres for protein drug delivery. Journal of the Royal Society Interface, 3, 573-582. doi:10.1098/rsif.2006.0120

[38] Di Martino, P., Joiris, E., Gobetto, R., Masic, A., Palmieri, G.F. and Martelli, S. (2004) Ketoprofen-poly (vinylpyrrolidone) physical interaction. Journal of Crystal Growth, 265, 302-308. doi:10.1208/pt0802037

[39] Manna, L., Banchero, M., Sola, D., Ferri, A., Ronchetti, S. and Sicardi, S. (2007) Impregnation of PVP microparticles with ketoprofen in the presence of supercritical $\mathrm{CO}_{2}$. The Journal of Supercritical Fluids, 42, 378-384. doi:10.1016/j.supflu.2006.12.002

[40] Sancin, P., Caputo, O., Cavallari, C., Passerini, N., Rodriguez, L., Cini, M. and Fini, A. (1999) Effects of ultrasound-assisted compaction on ketoprofen/Eudragit S100 mixtures. European Journal of Pharmaceutical Sciences, 7, 207-213. doi:10.1016/S0928-0987(98)00022-0

[41] Rawlinson, C.F., Williams, A.C., Timmins, P. and Grimsey, I. (2007) Polymer-mediated disruption of drug crystallinity. International Journal of Pharmaceutics, 336, 4248. doi:10.1016/j.ijpharm.2006.11.029

[42] Merisko-Liversidge, E., Liversidge, G.G. and Cooper, E.R. (2003) Nanosizing: A formulation approach for poorlywater-soluble compounds. European Journal of Pharmaceutical Sciences, 18, 113-120. doi:10.1016/S0928-0987(02)00251-8

[43] Demir, M.M. (2010) Investigation on glassy skin formation of porous polystyrene fibers electrospun from DMF. eXPRESS Polymer Letters, 4, 2-8.

[44] Kumari, A., Yadav, S.K. and Yadav, S.C. (2010) Biodegradable polymeric nanoparticles based drug delivery systems. Colloids and Surfaces B: Biointerfaces, 75, 1-18. doi:10.1016/j.colsurfb.2009.09.001 